\title{
Developpement larvaire du Serpulien cavernicole Marifugia cavatica Absolon et Hrabe (Polychaeta, Sedentaria)
}

\author{
Par Janez Matjašič et Boris Sket ${ }^{1}$ )
}

Avec Planches 3 (1) et 4 (2)

\section{Introduction}

Au cours de l'expédition spéléologique faite en septembre 1963 par l'Institut de Biologie de l'Université de Ljubljana, nous avons trouvé en Herzégovine pour la première fois de nombreuses larves du Polychète Serpulien d'eau douce cavernicole Marifugia casatica Absolon et $\left.H_{r a b e}{ }^{2}\right)$. Bien que quinze jours avant notre arrivée les gouffres absorbants du bas du Popovo polje étaient encore remplis d'eau, nous avons trouvé à sec la grande quantité des petits tubes qui croissent sur les parois de ces gouffres; mais nous avons pu constater qu'en prélevant un morceau de la masse formée par les tubes de ce Polychète sédentaire et en le mettant ensuite dans un récipient avec de l'eau beaucoup de larves apparaissaient rapidement. Les recherches ultérieures ont été faites au Laboratoire de l'Institut de Ljubljana oú nous élevons les animaux cavernicoles dans une cave où la température de l'eau est en été de 13 à 15 degrés C, ce qui correspond aux conditions du sous-sol du Popovo polje.

Le développement depuis la trochophore jusqu'au stade où apparaissent les ébauches de collerette a duré deux jours en moyenne, et malheureusement nous n'avons pas pu suivre le développement ultérieur. Mais vers la fin décembre, nous avons aperçu sur les parois de l'aquarium dans lequel un bloc de tubes de Marifugia était immergé, des exemplaires juvéniles avec leurs tubes et leurs tentacules digitiformes. Jusqu'à la moitié de février, ceux-ci n'ont point changé morphologiquement et ne se sont pas développés, puis ils ont peu à peu disparu.

1) Institut za Biologijo, Univerze v. Ljubljani, Ljubljana (Yougoslavie).

$\left.{ }^{2}\right)$ M. Dizdarevič a récolté en janvier et février 1963 cinq métatrochophores de Marifugia dans un aquarium du laboratoire où des blocs de cette Annélide étaient conservés depuis une année. 


\section{Morphologie externe}

La description du développement que nous présentons ici est encore assez incomplète. Quelques stades de développement manquent et, pour quelques autres, nous n'avons trouvé que très peu d'exemplaires. Mais on doit souligner le fait que l'on rencontre fréquemment des formes anormales et que le cours habituel du développement est souvent perturbé, ainsi que nous avons pu le constater parmi les exemplaires juvéniles provenant de diverses grottes.

Le dimorphisme sexuel des sujets adultes ne se montre que par la couleur. Les gonades, logées dans la partie caudale de l'abdomen, se vident par les fentes transversales de la paroi du corps. Le sperme est typique, les ovules ronds, hololécithiques, incolores (fig. A). La manière et le lieu de la fécondation ne sont pas encore connus, mais il est certain que l'opercule ne prend pas part à l'acte de reproduction.

\section{Pré-trochophore}

Le stade le plus jeune que nous ayons observé était celui de la blastula ou de la gastrula, avec la forme d'une petite sphère dont toute la surface était couverte de cils. Elle se mouvait comme une trochophore, mais plus lentement.

\section{Trochophore et métatrochophore}

L'épisphère est arrondie, tandis que l'hyposphère est allongée et ordinairement un peu incurvée. Près de la bouche, deux plis semicirculaires se sont développés (fig. B).

La ciliature est composée d'une touffe de longs cils vibratiles sur la plaque sincipitale et du prototroche, mais on aperçoit aussi des cils vibratiles très petits qui recouvrent de petites surfaces autour de la bouche et vers le pôle anal (probablement aussi autour de l'anus même).

La trochophore reste habituellement entre les tubes, mais vient aussi dans les tubes des Marifuges adultes ; le reste du temps elle nage, en suivant une ligne hélicoïdale, à l'aide de ses couronnes ciliaires.

Par sa forme et sa ciliature, le premier stade de la métatrochophore ne diffère presque pas de la trochophore, sauf que l'hyposphère est en général trois fois étranglée en forme d'anneau, et qu'on aperçoit déjà, au côté dorsal, deux paires de soies séparées et légèrement recourbées (fig. C).

Dans son développement ultérieur, la larve continue de s'allonger et la troisième paire de soies se développe (fig. $\mathrm{D}$ et $\mathrm{D}_{\mathbf{1}}$ ). 


\section{Nectochaeta avec collerette}

Bientôt le corps de la larve se creuse profondément au dessous du prototroche. La partie antérieure de la larve (= céphalon), séparée par cette rainure, a la forme d'une poire fortement aplatie à la base et au sommet, de sorte qu'elle est plus large que haute. Sous cette rainure, une collerette étroite commence à se former, de sorte que deux plis transversaux, dirigés vers l'anus, se produisent des deux côtés de la ligne médiane au côté ventral. Ces plis ne sont pas encore unis sur la ligne médiane et ils ne passent pas encore au côté dorsal, ils se disjoignent sur les côtés. Le reste du corps comporte, comme au stade initial de nectochaeta, trois paires de soies (fig. E).

\section{Nectochaeta avec «col»}

Le stade suivant se distingue par une espèce de col qui s'est développé entre la tête piriforme et la collerette ; ce col, dont le diamètre est plus petit que celui de l'épisphère, est séparé de la partie apicale du corps par une rainure circulaire. La collerette s'est tant développée que son bord postérieur atteint presque la base de la première paire de soies (qui se situe d'ailleurs du côté opposé). Au pôle anal de la larve, une paire de petites plaques semi-circulaires dentelées (uncini) s'est formée (fig. F et U).

Dans ces deux stades, il y a un anneau de cils plus forts qui se trouve dans la rainure au dessus de la collerette, mais on ne l'aperçoit plus dans les coupes histologiques.

Il est vrai que les larves de ces stades sont encore capables de se déplacer en nageant, comme les larves plus jeunes, mais ordinairement elles reposent déjà sur le fond, légèrement fixées par une secrétion dans laquelle des débris étrangers se collent aussi.

\section{Nectochaeta avec ébauche de tentacules}

Le céphalon (épisphère + partie basale de l'hyposphère) qui était antérieurement piriforme, développe latéralement une paire de languettes bilobées qui donneront les tentacules. Subterminalement, au contact des deux languettes, se trouve une boulette de cellules, représentant probablement les rudiments de l'organe sincipital (fig. G).

La collerette n'a pas encore subi de changements considérables. Chaque sétigère semble ne porter encore qu'une seule soie de chaque côté. Les uncini de la partie anale du corps ont par contre doublé leur nombre, et surtout les nouveaux qui sont au côté ventral ont plutôt une forme triangulaire. Il y a latéralement, entre les dernières soies et 
les uncini subanaux, deux rainures transversales, et le champ périanal forme une coupole prononcée qui surplombe les petites plaques déjà mentionnées (fig. $G$ et $G_{1}$ ).

On aperçoit des cils forts dans la rainure circulaire au-dessus de la collerette et dans la rainure qui sépare les deux lobes de chaque languette; dans ce dernier cas, il s'agit probablement de l'ancien prototroche.

La larve ainsi constituée ne nage plus (bien qu'elle soit encore capable de le faire) ; elle reste couchée ou collée au substratum par le côté dorsal.

\section{Stade tubicole à tentacules digitiformes}

Les languettes de la tête se sont développées en trois paires de tentacules simples et digitiformes; la paire ventrale est la plus courte, les paires dorsales portent déjà souvent médio-basalement les ébauches de tentacules nouveaux. Un opercule en forme de massue s'est développé au côté dorsal du groupe gauche de tentacules. Tout d'abord il est plus court et un petit peu plus gros que les tentacules, et il est apiculairement arrondi (fig. $\mathrm{H}$ ) ; quand il se développe complètement, le pédoncule seul atteint presque la longueur des tentacules et la partie élargie se redresse apiculairement. La boulette de cellules au contact des deux tentacules dorsaux est souvent encore visible (fig. $J$ et $J_{1}$ ).

La collerette s'est tellement développée ventralement et dorsalement que les deux premières paires de soies préexistantes y sont maintenant incluses. En arrière de la collerette le corps s'est allongé considérablement. La première paire d'uncini s'est développée un peu au dessous des soies thoraciques et, dans le groupe subanal, trois petites plaques du même genre se sont développées de chaque côté. A part celles-ci, l'abdomen possède aussi la dernière paire de soies préexistante, juste sous la collerette.

Il y a de longs cils vibratiles sur toute la partie supérieure des tentacules et des cils courts autour de l'anus.

Le tube, incomplètement bâti du côté du substratum, reste à moitié ouvert vers la base. Chez quelques individus il est droit, ailleurs il est très courbé. L'incrustation calcaire est encore très faible, c'est pourquoi la paroi du tube est diaphane et légèrement élastique; la structure est finement granulée, on aperçoit déjà des bandes annulaires. La longueur du tube est environ d'1 mm.

D'habitude, l'animal se tient dans le tube de telle manière que la couronne de tentacules et le bord supérieur de la collerette en émergent. La partie abdominale du corps est très allongée et amincie, sauf 
juste avant l'anus oú elle s'élargit, de sorte qu'elle s'appuie sur la pairo du tube vers son milieu, avec les uncini dentelés (fig. J). Les cils vibratiles des tentacules battent sans cesse. Après chaque excitation l'animal se contracte aussitôt, se retire dans le tube, et s'y enfonce profondément.

\section{Stade tubicole à tentacules penniformes}

Un peu plus tard, les tentacules acquièrent des pinnules latérales. A la place des deux paires de soies doubles de la collerette, des petites touffes se développent, composées de 4 à 5 soies peu courbées (fig. CH), et près de celles-ci les uncini se multiplient considérablement. A ce stade, l'abdomen est déjà bien développé, formé de plusieurs segments portant chacun des touffes de soies géniculées, ainsi que plusieurs petites plaques dentelées.

Le tube est entièrement calcifié, très allongé, mais mince et sans sculpture évidente.

\section{Morphologie interne}

Les cellules qui portent les cils vibratiles du prototroche sont particulièrement vacuolisées. La plaque sincipitale de la trochophore est très bien développée et, même chez les métatrochophores et chez les nectochaetae, elle est munie d'une longue touffe de cils vibratiles. Les cellules de l'organe sincipital représentent un épaississement ectodermique du sommet de l'épisphère qui s'étend sur la voûte vers le côté ventral. L'organe sincipital peut se rétracter légèrement sous l'action de muscles spéciaux attachés à lui. Nous n'avons trouvé aucun autre organe de sens; seule la touffe de cils vibratiles qui termine le pygidium doit être en rapport avec un certain sens. Il n'y a aucune trace d'yeux. Probablement le développement du système nerveux ne diffère pas essentiellement de celui qu'on connaît chez les autres Polychètes. Parmi les nerfs larvaires, l'anneau nerveux sous le prototroche est très bien développé.

La cavité générale primaire (blastocoele) est relativement petite à cause du grand développement du tube digestif et des filaments mésodermiques qui la traversent en tous sens.

Hors la musculature larvaire d'origine mésenchymateuse (musculature de la tête), la nectochaeta acquiert aussi la musculature du tronc qui nait des filaments mésodermiques. Chez la nectochaeta, on aperçoit déjà la musculature qui sert au battement des soies.

Le tube digestif de la trochophore et de la nectochaeta est typique. La bouche est large, mais pas autant que celle de Mercierella. Elle con- 
duit à un œsophage fortement cilié. Le lumen de l'estomac n'est pas si vaste que celui de beaucoup de larves de Polychètes. Le côlon conduit au rectum qui se vide subterminalement. Le côlon et le rectum sont aussi pourvus de cils vibratiles. Les cellules de l'estomac sont extrêmement volumineuses et pleines de grosses sphérules. Le tube digestif monte d'abord vers l'organe sincipital, puis il décrit, comme chez la larve de Mercierella (Rullier, 1955), quelques spirales. Enfin il descend presque en ligne droite vers l'anus. La nectochaeta plus âgée a déjà un tube digestif mieux formé. Chez la trochophore, les deux ceintures mésodermiques apparaissent très tôt. Elles montent haut vers l'épisphère. Tout d'abord elles sont séparées, elles se rapprochent beaucoup vers le milieu de l'hyposphère, de sorte qu'on aperçoit tout juste la limite entre elles, ensuite elles s'éloignent un peu de nouveau. Un peu avant le stade de nectochaeta, trois paires de sacs coelomiques se forment (stade métatrochophore). C'est ainsi que naissent les trois premiers segments larvaires qui sont caractéristiques pour le genre Nereis et pour les Serpulidae (Dawydoff, 1959). Ces trois segments restent très longtemps les seuls qu'on voit dans le développement. Les segments postlarvaires qui se forment ensuite proviennent probablement de la zone de prolifération de la région postéro-terminale.

Les protonéphridies, d'abord simples, sont suivies plus tard par une deuxième paire semblable à la première.

\section{Conclusions}

Le développement de la Marifugia a montré que celle-ci, bien qu'elle soit depuis longtemps un animal d'eau douce et cavernicole, a conservé le développement larvaire qui est caractéristique pour les organismes marins. Sa trochophore est tellement typique qu'elle ne diffère pas, de manière essentielle, des trochophores de ses parents marins. Rien en elle n'indique quelque changement causé par sa vie dans l'eau douce. A part le manque d'une ébauche des yeux, on ne voit aucune conséquence de la vie souterraine. Chez les autres Polychètes d'eau douce, tels que Hypaniola kowalewskii (Grimm) et Manayunkia caspica Ann. du Danube, ainsi que Manayunkia baicalensis (Nusb.), il n'y a pas de stade larvaire libre (mais nous ne savons pas si on a fait des recherches sur les parents marins les plus proches de ces espèces!). Les larves qui restent dans les tubes maternels ne sont pas du type «trochophore» (Popescu-Marinescu, 1964; Kožov, 1962).

La plupart des animaux d'eau douce qui sont d'origine marine, mais qui vivent depuis longtemps dans l'eau douce, ont perdu le développement post-embryonnaire qui est caractéristique pour tous leurs parents 
marins. Mais les spécimens qui sont parvenus en eau douce récemment ont conservé les stades larvaires marins (par. ex. : Dreissensia).

Probablement déjà dans le tertiaire, la Marifugia vivait dans les eaux douces à la surface de la terre, d'oú elle e'st retirée plus tard sous terre, comme quelques autres animaux d'eau douce, à cause de certaines modifications géologiques et hydrographiques.

La théorie de son passage direct de la mer Adriatique dans les cours d'eau souterrains n'est pas établie, étant donné que la Marifugia est représentée aussi dans le système fluvial de la mer Noire ; les conditions qu'auraient rencontré les autres groupes animaux faisant le mème voyage s'y opposent aussi.

A propos de la durée du développement des divers stades, on pourrait dire qu'elle est très variable et que parfois le passage depuis la trochophore jusqu'à la nectochaeta avancée peut être très bref (il peut durer un seul ou deux jours). Quant au développement des sujets jeunes qui sont déjà dans les tubes, il est très lent.

On dirait que l'ébauche des tentacules ne provient pas de l'épisphère seule, mais aussi de l'hyposphère. C'est que les restes du prototroche se trouvent entre les deux rameaux de chaque ébauche de tentacules.

\section{RÉSUMÉ}

Parmi le matériel venant de l'Herzégovine, on a trouvé des trochophores vivantes du Serpulien cavernicole Marifugia cavatica. Dans le laboratoire de l'Institut de Biologie de l'Université de Ljubljana, on a observé le développement ultérieur qui passe par les stades de métatrochophore et de nectochaeta. La constitution de la trochophore est typique. La nectochaeta possède trois métamères et chaque métamère une seule paire de soies. La nectochaeta une fois descendue au fond, la collerette et les ébauches des tentacules commencent à se développer. Le tube, trois paires de tentacules simples et l'opercule sont formés aux stades suivants. Tant que le tube est court et complètement adhérent, l'animal repose collé au substratum par son côté dorsal.

\section{ZUSAMMENFASS UNG}

Im Material aus der Herzegowina wurden lebende Trochophoren der Höhlen-Serpulide Marifugia cavatica gefunden. Im Laboratorium des Biologischen Instituts der Universität in Ljubljana wurde die weitere Entwicklung beobachtet, die über die Metatrochophora- und Nectochaeta-Stadien verläuft. Die Trochophora ist typisch gebaut. Nectochaeta hat drei Metameren, jede Metamere nur ein Paar Borsten. Nachdem sich die Nectochaeta an die Unterlage gesetzt hat, beginnt die Entwicklung des Kragens und der Tentalelanlagen. Die nachfolgenden Stadien haben eine Röhre, drei Paar einfache Tentakel und ein Operculum. Solange die Röhre noch kurz und anliegend ist, liegt das Tier mit der Rückenseite auf Unterlage. 


\section{B I B LIOGRAP H I E}

Absolon, K., et Hrabě, S. (1937) - Über einen neuen Süßwasser-Polychaeten aus den Höhlengewässern der Herzegowina. - Zool. Anz., 88, H. 9/10: 249-264.

Dawydoff, C. (1959) - Ontogenèse des Annélides. - Traité de Zoologie, T. 5, f. 1: 594-686. Paris.

Dizdarević, M. (1964) - The larvae of fresh-water cave polychaete Marifugia cavatica Absolon et Hrabě. - Bull. Scient. Conseil Acad. RSF Yougoslavie, 9, no 1/2: 10.

Fischer-Piette, E. (1937) - Sur la biologie du Serpulien d'eau saumâtre Mercierella enigmatica Fauvel. - Bull. Soc. Zool. France, LXII, no 3 : 197-208.

Hempelmann, F. (1928-1934) - Archiannelida und Polychaeta. - Kükenthal, Handbuch Zool. II.

Kožov, M. M. (1962) - Biologija ozera Bajkal, Moskva.

Popescu Marinescu, V. (1964) - La reproduction et le développement des Polychètes reliques ponto-caspiens du Danube: Hypaniola koxpaleswskii (Grimm) et Manayunkia caspica Ann. - Rev. Roumaine Biol., Ser. Zool., 9, no 2: 87-100.

Rullier, F. (1955) - Développement du Serpulien Mercierella enigmatica Fauvel. - Vie et Milieu, 6, no $2: 225-240$.

Vuillemin, S. (1954-1955) - Formation de la trochophore de Mercierella enigmatica Fauvel. - Bull. Soc. Sci. Nat. Tunisie, 7, no 1-4: 193-194.

\section{EXPLICATION DES PLANCHES 3 (1) et 4 (2)}

Marifugia cavatica Absolon et Hrabě : partie de l'ontogenèse. A, œuf vierge; $\mathrm{B}$, trochophore - métatrochophore (latéralement); $\mathrm{C}-\mathrm{D}$, nectochaeta (côté dorsal et latéral); E, nectochaeta avec ébauche de collerette (côté ventral); F, nectochaeta avec «col» (côté dorsal); G, larve avec ébauche de couronne brachiale $=$ avec «voiles» (côté dorsal et ventral) $; \mathrm{H}$, jeune Marifugia avec ébauche d'opercule; I, jeune Marifugia à opercule développé (côté dorsal et ventral ; l'orientation écologique est inverse! La partie inférieure et ouverte du tube est fixée à la base).

$\mathrm{b}$, bouche; $\mathrm{c}$, collerette; ch, chetae; $\mathrm{u}$, uncini ; t, rudiment de la plaque sincipitale (?) ; a, anus; br, ébauche de la couronne brachiale; op, opercule. 
SPELEOLOGY II

(Matjasic \& Sket, 1)

PLATE 3

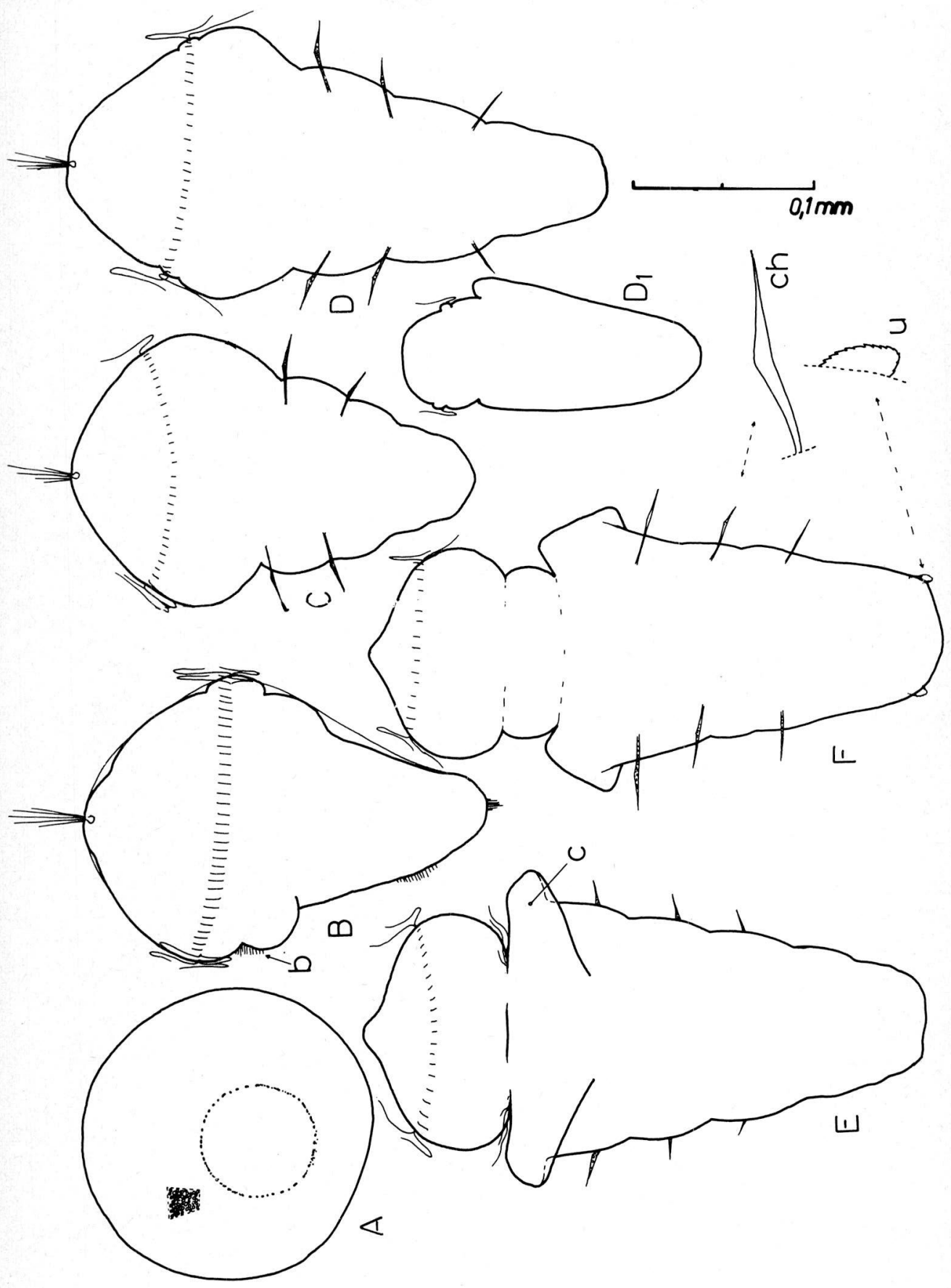




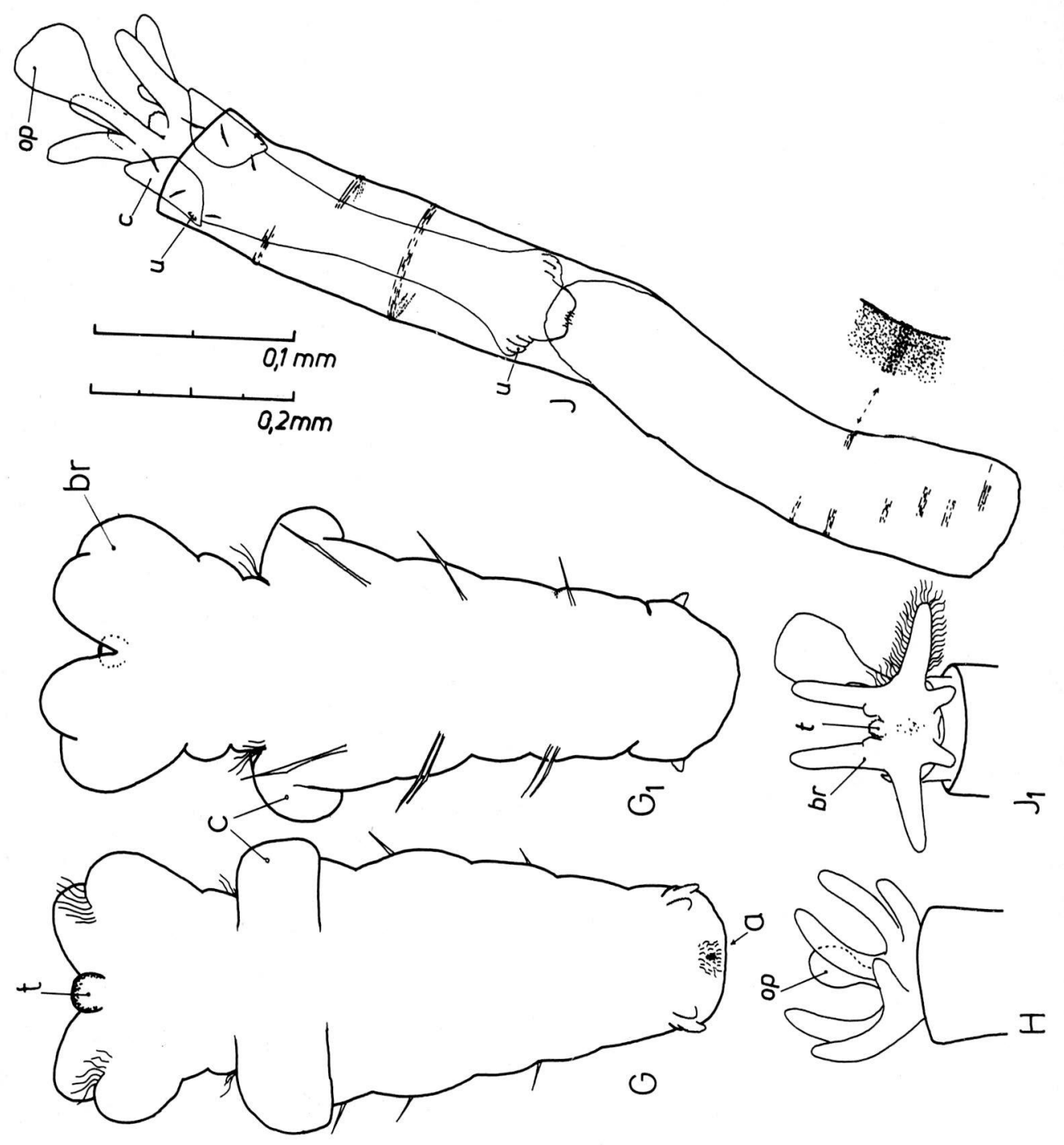

